\title{
Мощные диоды Шоттки с участком отрицательного дифференциального сопротивления на вольт-амперной характеристике
}

\author{
(C) А.Г. Тандоев, Т.Т. Мнацаканов , С.Н. Юрков \\ Национальный исследовательский университет „Московский энергетический институт“, \\ 111250 Москва, Россия \\ ฯ E-mail: mnatt@yandex.ru
}

Поступила в Редакцию 9 сентября 2020 г.

В окончательной редакции 14 сентября 2020 г.

Принята к публикации 18 сентября 2020 г.

Показано, что при больших плотностях тока, превышающих некоторую величину $j_{\mathrm{st} 1}$, в базовой области диодов Шоттки наряду с диффузионным режимом переноса осуществляется так же режим диффузии, стимулированной квазинейтральным дрейфом. Было исследовано влияния этого недавно обнаруженного режима на вид вольт-амперных характеристик диодов Шоттки при больших плотностях тока. Показано, что в случае, когда величина отношения ширины базовой области к амбиполярной диффузионной длине становится больше единицы, на вольт-амперной характеристике появляется участок с отрицательным дифференциальным сопротивлением. Результаты аналитического исследования были проверены и подтверждены с помощью численного моделирования.

Ключевые слова: режимы переноса носителей заряда в полупроводниках, мощные диоды Шоттки, вольт-амперная характеристика, влияние режимов переноса носителей на характеристики мощных структур.

DOI: $10.21883 /$ FTP.2021.01.50390.9521

\section{1. Введение}

В настоящее время диоды Шоттки (ДШ) являются одними из наиболее распространенных приборов полупроводниковой электроники. Преимущества ДШ перед приборами с $p-n$-переходами по ряду важных динамических параметров - быстродействию, коммутационным потерям и т.д. обусловлены отсутствием накопления неравновесных носителей заряда в базовой области в рабочих режимах протекания прямого тока. Однако в аварийных режимах (в режиме короткого замыкания или в режиме ударного тока) плотность прямого тока может в десятки раз превосходить плотность тока в рабочем режиме [1-3]. В работах [4-6] отмечалась возможность инжекции неосновных носителей заряда контактом Шоттки и протекание прямого тока в режиме высокого уровня инжекции (ВУИ) в базовой области [4-6]. Наиболее полное исследование протекания прямого тока в условиях ВУИ, проведенное в рамках диффузионного приближения, представлено в работе [7].

В работах $[8,9]$ были рассмотрены вольт-амперные характеристики (BAX) диодных структур с $p$-n-переходами при плотностях токов, когда в базовой области $n$-типа осуществлялся режим ВУИ. Было показано, что если отношение $W_{n} / L\left(W_{n}\right.$ - толщина базового слоя, $L-$ амбиполярная диффузионная длина) достигает величины $\sim 8-10$, то наряду с известными квазинейтральными режимами переноса (диффузионным и дрейфовым) необходимо учитывать режим диффузии, стимулированной квазинейтральным дрейфом (DSQD mode), и было исследовано влияние этого режима на вид прямой ВАХ. В работе [8] было показано, что режим DSQD проявляется и при малой величине отношения $W_{n} / L$, если распределение концентрации носителей заряда сильно перекошено. Такая ситуация характерна для ДШ, у которых наблюдается существенная асимметрия инжектирующих способностей переходов $\mathrm{Me}-п / п$ (металл-полупроводник) и $n-n^{+}$.

В работах $[10,11]$ был представлен аналитический расчет ВАХ ДШ для структур, у которых отношение $W_{n} / L$ было меньше единицы, распределение носителей заряда монотонно возрастало, а во всей базовой области осуществлялся режим ВУИ. Было показано, что влияние области DSQD приводит к парадоксальной зависимости падения напряжения от уровня легирования базовой области $N_{d}$ : с ростом $N_{d}$ падение напряжения увеличивалось.

Недавно в работе [12] был представлен новый механизм формирования $S$-образной ВАХ ДШ при плотностях тока, когда во всей базовой области осуществлялся ВУИ. Если отношение $W_{n} / L$ в результате, например, радиационного воздействия возрастало и становилось больше единицы, то распределение неравновесных носителей заряда переставало быть монотонным, и на распределении появлялся минимум. Слева от точки минимума производная $d p / d x$ отрицательна и перенос в этой области осуществляется в режиме квазинейтрального дрейфа. С ростом плотности тока точка минимума сдвигалась в сторону перехода Ме-п/п, и при достижении некоторой величины $J_{\mathrm{st}}$, вычисленной в работе [12], „слипалась“ с ним. При этом распределение неравновесных носителей заряда становилось монотонным, а область, в которой перенос осуществлялся в режиме 
квазинейтрального дрейфа, исчезала. Это приводило к перестроению квазинейтрального электрического поля и определяло $S$-образный вид ВАХ ДШ.

В настоящей работе продолжено исследование ВАХ ДШ при плотностях тока, превосходящих величину $J_{\text {st. }}$. Результаты аналитического исследования будут проверены и подтверждены с помощью численного моделирования.

\section{2. Постановка задачи}

Рассмотрим ДШ с базовой областью $n$-типа, у которой отношение $W_{n} / L$ больше единицы (например $\left.W_{n} / L \approx 1.5\right)$. Будем полагать, что плотность прямого тока превышает величину $J_{\text {st }}$ и во всей базовой области реализуется ВУИ.

На рис. 1 схематично представлена рассматриваемая структура. Начало координат $x=0$ выбрано на границе квазинейтральной части базы и области пространственного заряда контакта Ме-п/п.

Для расчета ВАХ ДШ необходимо так же, как в работах [10-12], решить уравнение непрерывности при ВУИ в базовом слое с граничными условиями:

$$
\begin{gathered}
\frac{d^{2} p}{d x^{2}}-\frac{j}{j_{d}}\left(1-\frac{j}{j_{\mathrm{cr}}}\right) \frac{N_{d}^{2}}{p^{2} L} \frac{d p}{d x}=\frac{p}{L^{2}} \\
\left.\frac{d p}{d x}\right|_{x=0}=-\frac{j}{2 q D_{p}}+\frac{j_{s 0}}{q D} \frac{p^{2}(0)}{n_{i}^{2}}-\frac{j}{j_{d}}\left(1-\frac{j}{j_{\mathrm{cr}}}\right) \frac{N_{d}^{2}}{p(0) L} \\
\left.\frac{d p}{d x}\right|_{x=W_{n}}=\frac{j}{2 q D_{n}}-\frac{j_{s p}}{q D} \frac{p^{2}\left(W_{n}\right)}{n_{i}^{2}}
\end{gathered}
$$

где $\quad j_{d}=\frac{2 q D_{p}(b+1) N_{d}}{L}, \quad j_{\text {cr }}=\frac{q\left(\mu_{n 0}+\mu_{p 0}\right) N_{d}}{F_{s n}^{-1}-F_{s p}^{-1}}, \quad L=$ $=\sqrt{\frac{2 b}{b+1} D_{p} \tau} \quad-\quad$ амбиполярная диффузионная длина, $\mu_{n 0}$ и $\mu_{p 0}-$ подвижности электронов и дырок в слабом электрическом поле, $F_{s n}$ и $F_{s p}-$ характерные значения электрического поля, при которых дрейфовые скорости электронов и дырок

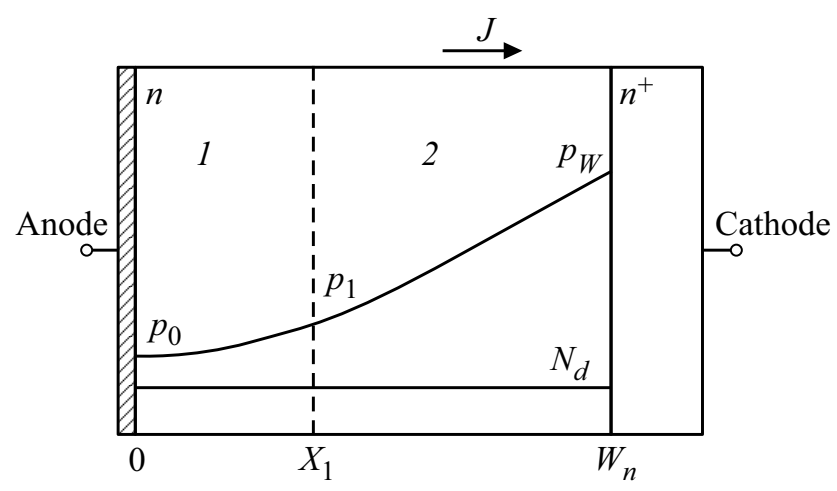

Рис. 1. Схематическое изображение ДШ и распределения концентрации неравновесных носителей заряда в базовой области ДШ в интервале плотностей тока $j$ от величины $j_{\text {st1 }}$ до $j_{\text {cr. }}$.

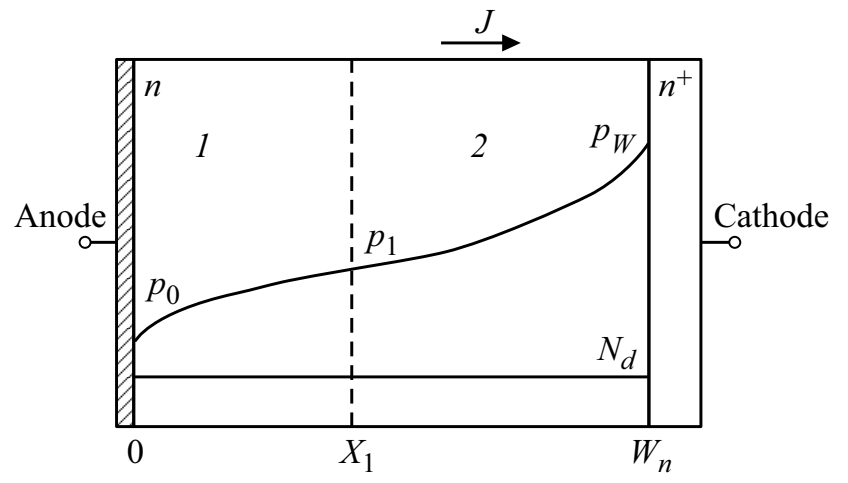

Рис. 2. Схематическое изображение ДШ и распределения концентрации неравновесных носителей заряда в базовой области ДШ при плотности тока $j$, превышающую величину $j_{\text {cr. }}$.

насыщаются, $j_{s 0}=A^{*} T^{2} \exp \left(-q \varphi_{B 0} / k T\right)-$ плотность тока насыщения перехода $\mathrm{Me}-\Pi / \Pi, A^{*}$ - эффективная постоянная Ричардсона, $j_{s p}-$ плотность тока насыщения $n^{+}-n$-перехода.

Сильная асимметрия инжектирующих способностей Ме-п/п- и $n-n^{+}$-переходов $\left(j_{s 0}\right.$ на $4-5$ порядков больше, чем $\left.j_{s p}\right)$ приводит к большому перекосу распределения неравновесных носителей заряда. Действительно, концентрация неравновесных носителей заряда вблизи перехода Ме-п/п $p(0)$ значительно меньше величины $p(W)$ - концентрации неравновесных носителей заряда вблизи $n-n^{+}$-перехода. Так как плотность тока превышает величину $J_{\text {st }}$, распределение носителей заряда монотонно возрастает от анодного перехода к катодному [12].

Если плотность тока $j$ меньше чем $j_{\text {cr }}$, то во всей базовой области, так же, как в работе [10], дрейфовый член отрицателен. В этом случае в базовой области образуется область DSQD, примыкающая к Me-п/п-переходу, обозначенная на рис. 1 цифрой 1 , и область диффузионного переноса, находящаяся между областью DSQD и $n-n^{+}$-переходом, которая обозначена на том же рисунке цифрой 2 .

Если же плотность тока превышает величину $j_{\mathrm{cr}}$, то во всей базовой области дрейфовый член положителен. В результате, так же, как и в работе [11], образуется область DSQD, в которой диффузионный член отрицателен. На рис. 2 эта область обозначена цифрой 1 , а область диффузионного переноса - цифрой 2.

Рассмотрим в начале диапазон плотностей тока от $J_{\mathrm{st}}$ до $j_{\mathrm{cr}}$. При некоторой плотности тока $J_{\mathrm{st} 1}$ область DSQD впервые выходит своей левой границей к переходу $\mathrm{Me}-\Pi /$ п. Существование области квазинейтрального дрейфового переноса при $j$ меньше чем $J_{\mathrm{st}}$, требует отрицательной величины производной $d p / d x$. (Обычно величина $J_{\text {st }}$ меньше, чем $j_{\text {cr. }}$.) Величина $J_{\text {st }}$ определялась в работе [12] из условия равенства нулю протяженности области с квазинейтральным дрейфовым переносом. С другой стороны, существование области DSQD требует положительной величины производной $d p / d x$. По 
этой причине величина плотности тока $J_{\mathrm{st} 1}$ оказывается больше, чем $J_{\text {st }}$, даже с учетом того, что применение метода региональных приближений [13] предполагает пренебрежение переходными областями. В дальнейшем величина плотности тока $J_{\mathrm{st1}}$ будет определена.

\section{3. ВАХ диода Шоттки при плотности тока в диапазоне от $j_{\text {st1 }}$ до $j_{\text {cr }}$}

Для нахождения приближенного решения нелинейного уравнения (1) воспользуемся, так же, как и в работах [10-12], методом региональных приближений [13].

Как было отмечено в предыдущем разделе, при плотности тока $J_{\mathrm{st} 1}$, область 1 (область DSQD) на рис. 1 впервые выходит своей левой границей к переходу Ме-п/п, распределение концентрации неравновесных носителей заряда становится монотонно возрастающим, а дрейфовый член в уравнении (1) во всей базовой области отрицательным. Так же, как и в работе [10], точка с координатой $x_{1}$ разбивает базовый слой ДШ на две области, обозначенные на рис. 1 цифрами 1 и 2 .

Низкая инжектирующая способность перехода Ме-п/п обусловливает сравнительно низкую концентрацию неравновесных носителей заряда в области 1. Поэтому в уравнении непрерывности (1) можно пренебречь членом, описывающим рекомбинационные потери, а само уравнение приобретает вид

$$
\frac{d^{2} p}{d x^{2}}-\frac{j}{j_{d}}\left(1-\frac{j}{j_{\mathrm{cr}}}\right) \frac{N_{d}^{2}}{p^{2} L} \frac{d p}{d x}=0 .
$$

Граничная концентрация неравновесных носителей заряда может быть получена с помощью граничного условия (2) и после несложных преобразований приобретает вид

$$
\begin{aligned}
p(0) & =p_{0} \\
& =n_{i} \sqrt{\frac{b}{b+1} \frac{j}{j_{s 0}}\left\{1+\frac{b+1}{b} \frac{q D p^{*}}{j L}\left[\left(\frac{p_{1}}{p^{*}}\right)^{3}+\frac{p^{*}}{p_{1}}\right]\right\}},
\end{aligned}
$$

где $p^{*}=N_{d} \sqrt{j / j_{d}\left(1-j / j_{\text {cr }}\right)}$ и $p_{1}=p\left(x_{1}\right)$.

Вторым граничным условием для уравнения (4), так же, как и в работе [12], является:

$$
\left.\frac{j}{j_{d}}\left(1-\frac{j}{j_{\mathrm{cr}}}\right) \frac{N_{d}^{2}}{p_{1}^{2} L} \frac{d p}{d x}\right|_{x_{1}}=\frac{p_{1}}{L^{2}} .
$$

Решение уравнения непрерывности в области 1 имеет вид

$$
\frac{p^{*}}{c L}\left(\frac{p-p_{0}}{p^{*}}+\frac{p^{*}}{c L} \ln \left|\frac{\frac{p}{p^{*}}-\frac{p^{*}}{c L}}{\frac{p_{0}}{p^{*}}-\frac{p^{*}}{c L}}\right|\right)=\frac{x}{L},
$$

где

$$
c=\frac{p^{*}}{L}\left[\left(\frac{p_{1}}{p^{*}}\right)^{3}+\frac{p^{*}}{p_{1}}\right] .
$$

Протяженность области $1 x_{1}$ определяется выражениями (7) и (8) при $p=p\left(x_{1}\right)=p_{1}$ :

$$
\begin{aligned}
& \frac{x_{1}}{L}=\frac{\left(\frac{p^{*}}{p_{1}}\right)^{2}}{1+\left(\frac{p^{*}}{p_{1}}\right)^{4}} \\
& \times\left\{1-\frac{p_{0}}{p_{1}}-\frac{\left(\frac{p^{*}}{p_{1}}\right)^{4}}{1+\left(\frac{p^{*}}{p_{1}}\right)^{4}} \ln \left|\frac{p_{0}}{p_{1}}\left[1+\left(\frac{p^{*}}{p_{1}}\right)^{4}\right]-\left(\frac{p^{*}}{p_{1}}\right)^{4}\right|\right\} .
\end{aligned}
$$

Область 2 на рис. 1 ограничена точками с координатами $x_{1}$ и $W_{n}$. Для этой области справедливы все результаты, полученные в работе [12]. Повторим их вкратце.

Высокая инжектирующая способность $n^{+}-n$-перехода обеспечивает большую концентрацию носителей заряда в области 2 и позволяет пренебречь в уравнении непрерывности (1) дрейфовым членом [13]. В результате в области 2 уравнение (1) приобретает диффузионный вид:

$$
\frac{d^{2} p}{d x^{2}}=\frac{p}{L^{2}}
$$

Общее решение уравнения (10), представляющее сумму нарастающей и спадающей экспонент, неудобно для дальнейшего анализа. Понижая порядок уравнения (10), так же, как в работе [12], приведем его к виду

$$
\frac{d p}{d x}=\sqrt{C_{1}+\left(\frac{p}{L}\right)^{2}} .
$$

Неизвестную константу $C_{1}$ определим, так же, как в работе [12], из условия (6), где производная $\left.(d p / d x)\right|_{x_{1}}$ определяется уравнением (11). В результате получим

$$
C_{1}=\left[\left(\frac{p_{1}}{p^{*}}\right)^{4}-1\right]\left(\frac{p_{1}}{L}\right)^{2}
$$

Решение уравнения (11) с граничным условием

$$
p\left(W_{n}\right)=p_{W}
$$

имеет вид

$$
\ln \frac{p_{W}+\sqrt{C_{1} L^{2}+p_{W}^{2}}}{p+\sqrt{C_{1} L^{2}+p^{2}}}=\frac{W_{n}-x}{L} .
$$

Выражения (12) и (14) позволяют определить протяженность области 2. Предполагая, что при $x=x_{1}$ $p=p_{1}$, получим

$$
\begin{aligned}
& \frac{W_{n}-x_{1}}{L} \\
& =\ln \left\{\frac{p_{W}}{p_{1}} \cdot \frac{\left(\frac{p^{*}}{p_{1}}\right)^{2}+\sqrt{\left[1-\left(\frac{p^{*}}{p_{1}}\right)^{4}\right]\left(\frac{p_{1}}{p_{W}}\right)^{2}+\left(\frac{p^{*}}{p_{1}}\right)^{4}}}{1+\left(\frac{p^{*}}{p_{1}}\right)^{2}}\right\} .
\end{aligned}
$$


Концентрация неравновесных носителей заряда вблизи $n^{+}-n$-перехода $p_{W}$ определяется с помощью граничного условия (3) и имеет стандартный вид $[10,11]$. В рассматриваемом диапазоне плотности тока (во всей базовой области реализуется ВУИ) величина $p_{W}$ хорошо аппроксимируется выражением

$$
p_{W}=n_{i} \sqrt{\frac{1}{b+1} \frac{j}{j_{s p}}} .
$$

Зависимость $p_{1}=p_{1}(j)$ определяется уравнением

$$
\frac{x_{1}}{L}+\frac{W_{n}-x_{1}}{L}=\frac{W_{n}}{L} \text {. }
$$

Протяженности областей 1 и 2, входящие в уравнение (17), заданы выражениями (9), (5), (15) и (16).

Найдем теперь плотность тока $J_{\text {st1 }}$, при которой область DSQD впервые соприкоснется своей левой границей с переходом Ме-п/п. При этом еще выполняется условие, связывающее концентрации неравновесных носителей в граничных точках области DSQD, полученное в работе [12]. Отметим, что при этом координата левой границы области DSQD становится равной нулю, а концентрация неравновесных носителей в этой точке $\mathrm{Me}-\Pi / п$ граничной концентрацией $p_{01}$ :

$$
\left(\frac{p_{1}}{p^{*}}\right)^{3}+\frac{p^{*}}{p_{1}} \approx \frac{p^{*}}{p_{01}} .
$$

Условие (18) позволяет связать величину $p_{1}$ с граничной концентрацией $p_{01}$ приближенным соотношением

$$
\frac{p_{1}}{p^{*}} \approx \frac{\left(\frac{p^{*}}{p_{01}}\right)^{1 / 3}}{1+\frac{1}{3}\left(\frac{p_{01}}{p^{*}}\right)^{4 / 3}} .
$$

Величина $p_{01}$ определяется, так же, как и в работе [12], выражением

$$
p_{01}=n_{i} \sqrt{\frac{b}{b+1} \frac{j}{j_{s 0}}} .
$$

Необходимо помнить, что выражения (18)-(20) справедливы только при плотности тока $j_{\mathrm{st} 1}$, когда область DSQD впервые соприкоснется своей левой границей с переходом Ме-п/п.

Величину плотности тока $j_{\text {st1 }}$ найдем как корень уравнения (17), в котором протяженность области 1 (9) и области 2 - (15) находились с учетом соотношения (19), а величины $p_{01}$ и $p_{W}$ определялись выражениями (20) и (16) соответственно.

Падение напряжения на ДШ определяется падением напряжения на областях 1,2 и падением напряжения на переходах $\mathrm{Me}-п / п$ и $n^{+}-n$. Найденные решения позволяют рассчитать все эти величины. Для областей 1 и 2 были получены следующие выражения:

$$
\begin{gathered}
U_{1}=\frac{2 b}{(b+1)^{2}} \frac{k T}{q} \frac{j L}{q D p^{*}} \frac{\left(\frac{p^{*}}{p_{1}}\right)^{3}}{1+\left(\frac{p^{*}}{p_{1}}\right)^{4}} \\
\times \ln \frac{1}{\left|\frac{p_{0}}{p_{1}}\left[1+\left(\frac{p^{*}}{p_{1}}\right)^{4}\right]-\left(\frac{p^{*}}{p_{1}}\right)^{4}\right|}, \quad(21) \\
U_{2}=\frac{2 b}{(b+1)^{2}} \frac{k T}{q} \frac{j L}{q D p^{*}} \frac{\left(\frac{p^{*}}{p_{1}}\right)^{3}}{\sqrt{1-\left(\frac{p^{*}}{p_{1}}\right)^{4}}} \\
\times \ln \frac{\sqrt{1-\left(\frac{p^{*}}{p_{1}}\right)^{4}}+1}{\sqrt{1-\left(\frac{p^{*}}{p_{1}}\right)^{4}} \frac{p_{1}}{p_{W}}+\sqrt{\left[1-\left(\frac{p^{*}}{p_{1}}\right)^{4}\right]\left(\frac{p_{1}}{p_{W}}\right)^{2}+\left(\frac{p^{*}}{p_{1}}\right)^{4}}} .
\end{gathered}
$$

Падение напряжения на переходах $\mathrm{Me}-\Pi / п$ и $n^{+}-n$ имеет стандартный вид:

$$
U_{\mathrm{Me} n n^{+}}=\frac{k T}{q} \ln \frac{p_{0} p_{W}}{n_{i}^{2}} .
$$

Полное падение напряжения на ДШ равно:

$$
U_{s h}=U_{1}+U_{2}+U_{\mathrm{Me} n n^{+}} .
$$

Рассмотрим случай, когда величина $L$ растет, а отношение $W_{n} / L$ становится меньше единицы. Учтем, что с ростом $L$ распределение концентрации неравновесных носителей заряда в базовой области становится близким к линейному, а величина производной $d p / d x$ перестает зависеть как от $x$, так и от $L$ и становится близкой к значению $p_{W} / W_{n}$, так как $p_{W} \gg p_{0}$. По этой причине величина $p_{1}$, как это следует из (6), растет с ростом $L$ при фиксированном значении плотности тока $j$ и при некотором значении $L$ начнет выполняться условие

$$
\left(\frac{p^{*}}{p_{1}}\right)^{4}<\left(\frac{p_{1}}{p_{W}}\right)^{2} \ll 1 .
$$

При выполнении условия (25) уравнение (17) упрощается и после несложных преобразований принимает вид

$$
\frac{p_{1}}{p^{*}} \approx\left(\frac{p_{W}}{p^{*}} \frac{L}{W_{n}}\right)^{1 / 3} .
$$

С учетом условия (25) и приближенного равенства (26) нетрудно показать, что падение напряжения на базовом слое ДШ $U_{W}=U_{1}+U_{2}$ становится равным

$$
U_{W}=\frac{2 b}{(b+1)^{2}} \frac{k T}{q} \frac{j W_{n}}{q D p_{W}} \ln \left|\frac{P_{W}}{p_{0}-p^{*}\left(\frac{p^{*}}{p_{1}}\right)^{3}}\right| .
$$

Полученные из более общих соображений соотношения (26) и (27) совпадают с выражениями, полученными ранее в работе [10] для ДШ, у которого отношение $W_{n} / L$ было меньше единицы. 


\section{4. ВАХ диода Шоттки при плотности тока, большей чем $j_{\text {cr }}$}

Как отмечалось выше, при плотности тока $j$, большей чем $j_{\text {cr }}$, дрейфовый член в уравнении (1) становится положительным во всей базовой области. Подобный случай для ДШ с величиной отношения $W_{n} / L$, меньшей единицы, был рассмотрен в работе [11]. Рассмотрим, так же, как и в предыдущих разделах, структуры с отношением $W_{n} / L$, превышающим единицу. Такая структура схематично изображена на рис. 2. Точка с координатой $x_{1}$, по аналогии с работой [11], разбивает базовую область на две части. В области, примыкающей к Ме-п/п-переходу, обозначенной на рис. 2 цифрой 1 , концентрация неравновесных носителей заряда сравнительно мала. Это позволяет так же, как и в предыдущем разделе, пренебречь рекомбинационным членом в уравнении непрерывности (1). В результате перенос носителей заряда в области 1 осуществляется в режиме DSQD. В отличие от случая, рассмотренного в разд. 3, дрейфовый член теперь положителен, диффузионный член становится отрицательным, а уравнение непрерывности (1) приобретает вид

$$
\frac{d^{2} p}{d x^{2}}+\frac{j}{j_{d}}\left(\frac{j}{j_{\mathrm{cr}}}-1\right) \frac{N_{d}^{2}}{p^{2} L} \frac{d p}{d x}=0 .
$$

Граничная концентрация неравновесных носителей заряда $p(0)$, так же, как в разд. 3 , получена с помощью граничного условия (2). Она отличается от выражения (5) и имеет вид

$$
\begin{aligned}
& p(0)=p_{0} \\
& =n_{i} \sqrt{\frac{b}{b+1} \frac{j}{j_{s 0}}\left\{1+\frac{b+1}{b} \frac{q D p_{1}^{*}}{j L}\left[\left(\frac{p_{1}}{p_{1}^{*}}\right)^{3}-\frac{p_{1}^{*}}{p_{1}}\right]\right\},},
\end{aligned}
$$

где

$$
p_{1}^{*}=N_{d} \sqrt{\frac{j}{j_{d}}\left(\frac{j}{j_{\mathrm{cr}}}-1\right)} .
$$

Вторым граничным условием для уравнения является очевидное соотношение

$$
\left.\frac{j}{j_{d}}\left(\frac{j}{j_{\mathrm{cr}}}-1\right) \frac{N_{d}^{2}}{p_{1}^{2} L} \frac{d p}{d x}\right|_{x_{1}}=\frac{p_{1}}{L^{2}} .
$$

Решение уравнения (28) с граничными условиями (29) и (31) отличается от полученного в разд. 3 решения (7) и имеет вид

$$
\frac{p_{1}^{*}}{c L}\left(\frac{p-p_{0}}{p_{1}^{*}}-\frac{p_{1}^{*}}{c L} \ln \left|\frac{\frac{p}{p_{1}^{*}}+\frac{p_{1}^{*}}{c L}}{\frac{p_{0}}{p_{1}^{*}}+\frac{p_{1}^{*}}{c L}}\right|\right)=\frac{x}{L},
$$

где

$$
c=\frac{p_{1}^{*}}{L}\left[\left(\frac{p_{1}}{p_{1}^{*}}\right)^{3}-\frac{p_{1}^{*}}{p_{1}}\right] .
$$

Протяженность области 1 также отличается от выражения, полученного в разд. 3:

$$
\begin{aligned}
\frac{x_{1}}{L}= & \frac{\left(\frac{p_{1}^{*}}{p_{1}}\right)^{2}}{1-\left(\frac{p_{1}^{*}}{p_{1}}\right)^{4}}\left\{1-\frac{p_{0}}{p_{1}}+\frac{\left(\frac{p_{1}^{*}}{p_{1}}\right)^{4}}{1-\left(\frac{p_{1}^{*}}{p_{1}}\right)^{4}}\right. \\
& \left.\times \ln \left|\frac{p_{0}}{p_{1}}\left[1-\left(\frac{p_{1}^{*}}{p_{1}}\right)^{4}\right]+\left(\frac{p_{1}^{*}}{p_{1}}\right)^{4}\right|\right\} .
\end{aligned}
$$

Рассмотрим теперь область 2, которая опять ограничена точками $x_{1}$ и $W_{n}$. Повторяя все рассуждения для области 2 приведенные в предыдущем разделе получим, что протяженность этой области определяется выражением $(15)$, в котором величина $p^{*}$ заменена на $p_{1}^{*}$ :

$$
\begin{aligned}
& \frac{W_{n}-x_{1}}{L} \\
& \quad=\ln \left\{\frac{p_{W}}{p_{1}} \frac{\left(\frac{p_{1}^{*}}{p_{1}}\right)^{2}+\sqrt{\left[1-\left(\frac{p_{1}^{*}}{p_{1}}\right)^{4}\right]\left(\frac{p_{1}}{p_{W}}\right)^{2}+\left(\frac{p_{1}^{*}}{p_{1}}\right)^{4}}}{1+\left(\frac{p_{1}^{*}}{p_{1}}\right)^{2}}\right\} .
\end{aligned}
$$

Величина $p_{W}$ определяется выражением (16). Зависимость $p_{1}=p_{1}(j)$ определяется, как и в разд. 3 уравнением (17), в котором протяженности областей 1 и 2 задаются соотношениями (34) и (35) соответственно.

Падение напряжения на всей структуре по-прежнему определяется падениями напряжений на областях 1,2 и на переходах. Вычислим величину падения напряжения $U_{1}$, используя решения уравнения непрерывности (32), (33):

$$
\begin{aligned}
U_{1}= & \frac{2 b}{(b+1)^{2}} \frac{k T}{q} \frac{j L}{q D p_{1}^{*}} \frac{\left(\frac{p_{1}^{*}}{p_{1}}\right)^{3}}{1-\left(\frac{p_{1}^{*}}{p_{1}}\right)^{4}} \\
& \times \ln \left|\frac{1}{\frac{p_{0}}{p_{1}}\left[1-\left(\frac{p_{1}^{*}}{p_{1}}\right)^{4}\right]+\left(\frac{p_{1}^{*}}{p_{1}}\right)^{4}}\right| .
\end{aligned}
$$

Падение напряжения на области 2 по форме совпадает с выражением (22), в котором величина $p^{*}$ заменена на $p_{1}^{*}$ :

$$
\begin{aligned}
& U_{2}=\frac{2 b}{(b+1)^{2}} \frac{k T}{q} \frac{j L}{q D p_{1}^{*}} \frac{\left(\frac{p_{1}^{*}}{p_{1}}\right)^{3}}{\sqrt{1-\left(\frac{p_{1}^{*}}{p_{1}}\right)^{4}}} \\
& \times \ln \frac{\sqrt{1-\left(\frac{p_{1}^{*}}{p_{1}}\right)^{4}}+1}{\sqrt{1-\left(\frac{p_{1}^{*}}{p_{1}}\right)^{4}} \frac{p_{1}}{p_{W}}+\sqrt{\left[1-\left(\frac{p_{1}^{*}}{p_{1}}\right)^{4}\right]\left(\frac{p_{1}}{p_{W}}\right)^{2}+\left(\frac{p_{1}^{*}}{p_{1}}\right)^{4}}} .
\end{aligned}
$$


Падение напряжения на переходах описывается соотношением (23), в котором величины $p_{0}$ и $p_{W}$ задаются выражениями (29) и (16) - соответственно.

Полное падение напряжения на ДШ описывается соотношением (24), в котором величины $U_{1}$ и $U_{2}$ задаются выражениями (36) и (37) соответственно, а падение напряжения на переходах - выражением (23).

Можно показать, проведя рассуждения по аналогии с разд. 3 , что в случае, когда величина отношения $W_{n} / L$ становится меньше единицы, уравнение (17) упрощается и принимает вид (26), в котором величина $p^{*}$ заменена на $p_{1}^{*}$. Падение напряжения на базовой области ДШ при этом становится равным

$$
U_{W}=\frac{2 b}{(b+1)^{2}} \frac{k T}{q} \frac{j W_{n}}{q D p_{W}} \ln \left|\frac{p_{W}}{p_{0}+p_{1}^{*}\left(\frac{p_{1}^{*}}{p_{1}}\right)^{3}}\right| .
$$

Выражение (38) совпадает с выражением, полученным в работе [11] для ДШ с величиной отношения $W_{n} / L$ меньше единицы.

\section{5. Обсуждение результатов и сравнение с численным экспериментом}

Рассматривался кремниевый ДШ со следующим набором параметров. Концентрация легирующей примеси в базовой области $n$-типа $N_{d}$ была равна $5 \cdot 10^{14} \mathrm{~cm}^{-3}$. Толщина базового слоя $W_{n}$ равнялась 100 мкм. Величина отношения $W_{n} / L$ была выбрана равной 1.5 . Плотность тока насыщения перехода $\mathrm{Me}-\Pi / \Pi j_{s}$ равнялась $1 \cdot 10^{-7} \mathrm{~A} / \mathrm{cm}^{2}$, а $n^{+}-n$-перехода $j_{s p}$ была равна $1 \cdot 10^{-12} \mathrm{~A} / \mathrm{cm}^{2}$. Такой же набор параметров был выбран при обсуждении результатов в работе [12]. Величина $j_{\text {cr }}$ при выбранном значении концентрации легирующей примеси $N_{d}$ была равна $1720 \mathrm{~A} / \mathrm{cm}^{2}$.

На рис. 3 представлены зависимости протяженности области 1 - области DSQD и области 2 - области диффузионного переноса от плотности тока $j$. Плотность прямого тока варьировалась от величины $j_{\mathrm{st} 1}$ до $2 \cdot j_{\mathrm{cr}}$. Для ДШ с рассматриваемым набором параметров величина $j_{\mathrm{st} 1}$, рассчитанная по методу, описанному в разд. 3, равнялась $520 \mathrm{~A} / \mathrm{cm}^{2}$. При приближении плотности тока к величине $j_{\text {cr }}$ протяженность области 1 уменьшалась и стремилась к нулю, а протяженность области 2 увеличивалась и стремилась к величине $W_{n}$. После того как плотность тока становилась больше чем $j_{\text {cr }}$, протяженность области 1 начинала монотонно возрастать, а области 2 - уменьшаться.

На рис. 4 представлена зависимость падения напряжения на областях 1,2 , суммарного падения напряжения на $\mathrm{Me}-п / п$ и $n^{+}-n$-переходах и полного падения напряжения на ДШ от плотности прямого тока $j$. Плотность прямого тока изменялась в том же диапазоне величин, как и на рис. 3. С ростом плотности тока $j$ и при

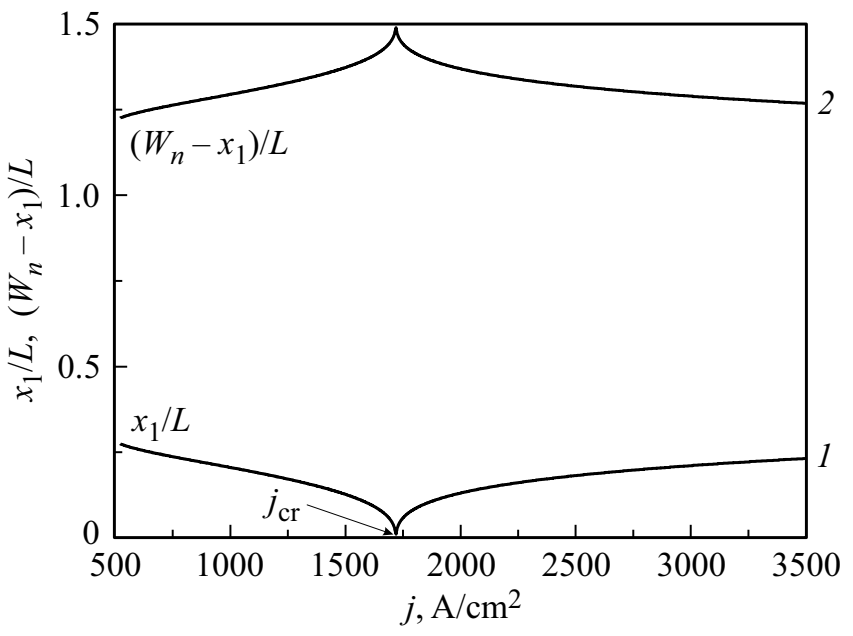

Рис. 3. Зависимость размера области DSQD (1) и области диффузионного переноса носителей заряда (2) от плотности тока $j$.

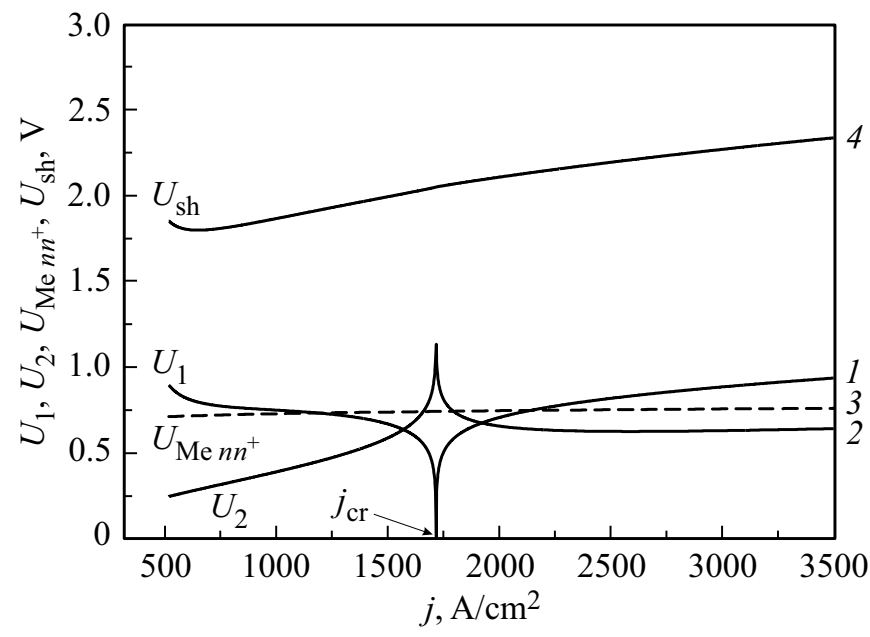

Pис. 4. Зависимость падения напряжения на области DSQD (1), области диффузионного переноса носителей заряда (2), суммарного падения напряжения на $\mathrm{Me}-\Pi / \Pi$ и $n^{+}-n$-переходах (3), полного падения напряжения на ДШ (4) от плотности тока $j$.

приближении к величине $j_{\text {cr }}$ падение напряжения на области $1 U_{1}$ уменьшалось и стремилаось к нулю, а падение напряжения на области $2 U_{2}$ увеличивалось. При дальнейшем росте, когда плотность тока становилась больше чем $j_{\text {cr, }}$, падение напряжения на области 1 начинало возрастать, а на области 2 сначала уменьшаться, а затем слабо возрастать. Падение напряжения на $\mathrm{Me}-\Pi / п$ и $n^{+}-n$-переходах во всем диапазоне плотностей тока изменялось логарифмически слабо. Немонотонная зависимость $U_{1}$ и $U_{2}$ от плотности тока $j$ приводила к немонотонной зависимости $U_{s h}-$ полного падения напряжения на ДШ. Следствием этого явилось появление на ВАХ ДШ участка с отрицательным дифференциальным сопротивлением $R_{d}$. 
Для проверки правильности модели было проведено сравнение результатов, полученных с помощью представленной модели, с результатами численного эксперимента. Расчет проводился с использованием программы INVESTIGATION (ISSLEDOVANIE). Эта же программа использовалась при проверке результатов в работе [12]. Подробное описание этой программы можно найти в работах $[14,15]$. Расчет проводился для кремниевого ДШ со следующим набором параметров. Толщина базового слоя $n$-типа $W_{n}$ была равна 100 мкм, толщина эмиттерного слоя $n^{+}$-типа равнялась 20 мкм. Концентрация донорной примеси в базовом слое $N_{d}$ была выбрана равной $5 \cdot 10^{14} \mathrm{~cm}^{-3}$, концентрация донорной примеси в эмиттере была равна $1 \cdot 10^{19} \mathrm{~cm}^{-3}$. Высота барьера Шоттки для электронов $\varphi_{B n}$ равнялась 0.85 эВ. Были выбраны следующие значения времен жизни неравновесных носителей заряда при ВУИ: $\tau=2.1$ (1), 2.8 (2), 3.8 (3), 5.4 (4), 8.5 (5), 15.1 мкс (6). Этим значениям соответствовали следующие величины отношения $W_{n} / L$ : $1-\frac{W_{n}}{L}=1.6,2-\frac{W_{n}}{L}=1.4,3-\frac{W_{n}}{L}=1.2,4-$ $\frac{W_{n}}{L}=1.0,5-\frac{W_{n}}{L}=0.8,6-\frac{W_{n}}{L}=0.6$.

Такой же набор параметров ДШ был использован при численной проверке аналитической модели, представленной в работе [12]. Результаты расчета ВАХ ДШ представлены на рис. 5. При увеличении времени жизни неравновесных носителей заряда $\tau$ и соответствующем уменьшении величины отношения $W_{n} / L \quad S$-образность BAX сначала уменьшалась, а затем пропадала совсем.

На рис. 6 приведены ВАХ ДШ, рассчитанные в соответствии с представленной в разд. 3 и 4 моделью. Расчет проводился для структур с тем же набором параметров для плотностей тока, превышающих значения $j_{\text {st1 }}$ (верхняя часть графиков ВАХ). Кроме того, на этом же рисунке приведены ВАХ ДШ, рассчитанные по аналитической модели, представленной в работе [12] (нижняя часть графиков ВАХ). Эти кривые были рассчитаны для плотностей тока, когда во всей базовой области был реализован ВУИ, но значение $j$ не превосходило введенную в работе [12] величину $j_{\text {st }}$. Сравнение рис. 5 и 6 показывает, что, несмотря на количественные различия, представленная модель качественно правильно описывает особенности ВАХ ДШ. Эти различия связаны с погрешностью метода региональных приближений [13]. При уменьшении величины отношения $W_{n} / L$ наблюдалось уменьшение $S$-образности BAX. Как видно из рис. 6, уменьшение величины отношения $W_{n} / L$ приводило к уменьшению величин плотностей тока $j_{\mathrm{st}}$ и $j_{\mathrm{st} 1}$. Физически это объясняется уменьшением рекомбинационных потерь неравновесных носителей заряда при уменьшении отношения $W_{n} / L$ и увеличением концентрации неравновесных носителей заряда вблизи Ме-п/п-перехода. Это приводит к уменьшению электрического поля, необходимого для поддержания в области, прилегающей к Ме-п/п-переходу, тока с требуемой плотностью $j$. Уменьшение отношения $W_{n} / L$ приводит к уменьшению протяженности области, в которой перенос носителей заряда осуществляется

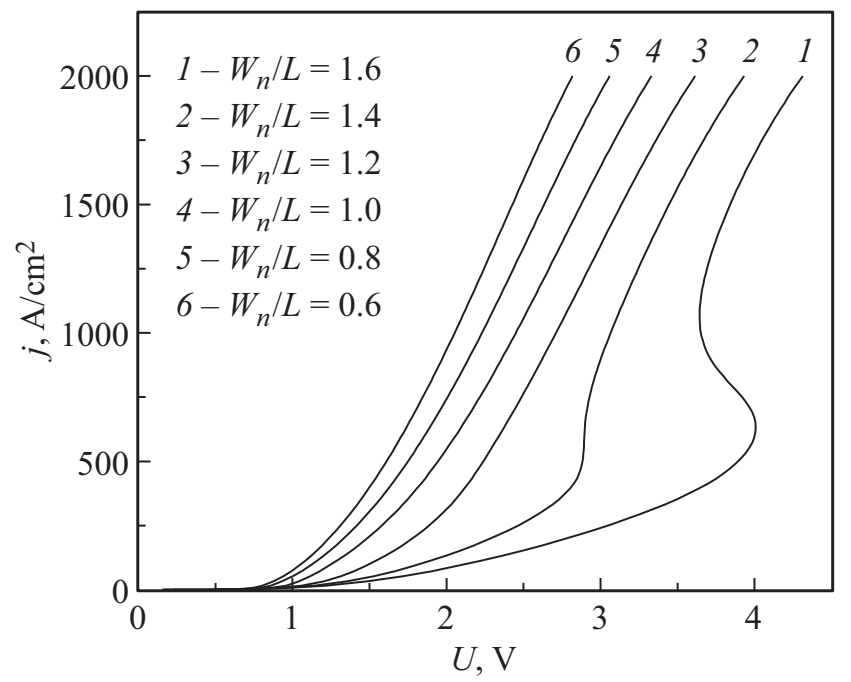

Рис. 5. Вольт-амперные характеристики ДШ, рассчитанные с помощью программы INVESTIGATION(ISSLEDOVANIE) для различных значений отношения ширины базовой области к амбиполярной диффузионной длине $W_{n} / L$, приведенных на рисунке.

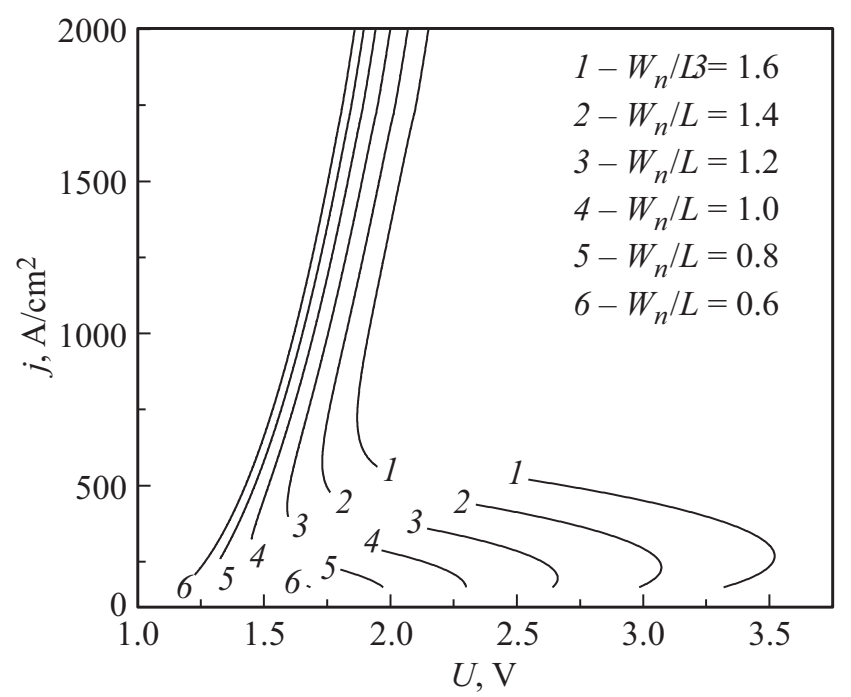

Рис. 6. Вольт-амперные характеристики ДШ, рассчитанные с помощью аналитической модели, представленных в данной работе и в работе [12]. Расчет проводился для различных значений отношения ширины базовой области к амбиполярной диффузионной длине $W_{n} / L$, приведенных на рисунке.

в режиме квазинейтрального дрейфа, рассмотренной в работе [12]. По этой причине уменьшается плотность тока $j_{\mathrm{st}}$, при котором происходит „схлопывание“ этой области и $j_{\mathrm{st} 1}$, при которой левая граница области DSQD впервые приближается к Ме-п/п-переходу.

\section{6. Заключение}

В работе было продолжено исследование ВАХ ДШ, начатое в работе [12]. Был рассмотрен случай, когда 
плотность тока превышала величину $j_{\mathrm{st} 1}$. В базовой области перенос носителей заряда осуществлялся в режиме ВУИ, а величина отношения $W_{n} / L$ принимала произвольные значения. При разработке модели было использовано граничное условие для перехода $\mathrm{Me}-п / п$, которое ранее успешно применялось в работах $[10,11]$. Это граничное условие учитывало слабую инжектирующую способность контакта Шоттки.

В работе было показано, что если величина отношения $W_{n} / L$ превышает единицу, то у ДШ наблюдается немонотонная зависимость падения напряжения от плотности тока. Это приводило к появлению на ВАХ ДШ области с отрицательным дифференциальным сопротивлением. При уменьшении величины отношения $W_{n} / L$ область на ВАХ с отрицательным дифференциальным сопротивлением уменьшалась и при некоторой величине отношения $W_{n} / L$ не возникала.

Результаты, полученные с помощью аналитической модели, были подтверждены численным экспериментом, выполненным с помощью программы INVESTIGATION (ISSLEDOVANIE).

\section{Финансирование работы}

Работа выполнена при поддержке Министерства образования и науки Российской Федерации (проект FSWF-2020-0022).

\section{Конфликт интересов}

Авторы заявляют, что у них нет конфликта интересов.

\section{Список литературы}

[1] L.M. Hillkirk. Solid State Electron., 48, 2181 (2004).

[2] M.E. Levinshtein, T.T. Mnatsakanov, P.A. Ivanov, J.W. Palmour, M.K. Das, B.A. Hull. Semicond. Sci. Technol., 23, 085011 (2008).

[3] J.W. Palmour, M.E. Levinshtein, P.A. Ivanov, Q.J. Zhang. J. Phys. D: Appl. Phys., 48, 235103 (2015).

[4] D.L. Scharfetter. Solid State Electron., 8, 299 (1965).

[5] A. Yu, E. Snow. Solid State Electron., 12, 155 (1969).

[6] B. Elfsten, P.A. Tove. Solid State Electron., 28, 721 (1985).

[7] W.T. Ng, S. Liang, C.A.T. Salama. Solid State Electron., 33, 39 (1990).

[8] T.T. Mnatsakanov, M.E. Levinshtein, A.G. Tandoev, S.N. Yurkov. J. Appl. Phys., 105, 044506 (2009).

[9] T.T. Mnatsakanov, A.G. Tandoev, M.E. Levinshtein, S.N. Yurkov. Semicond. Sci. Technol., 24, 075006 (2009).

[10] T.T. Mnatsakanov, M.E. Levinshtein, A.G. Tandoev, S.N. Yurkov, J.W. Palmour. Solid State Electron., 121, 41 (2016).

[11] T.T. Mnatsakanov, A.G. Tandoev, M.E. Levinshtein, S.N. Yurkov, J.W. Palmour. Phys. Semicond. Dev., 51, 8 (2017).

[12] А.Г. Тандоев, Т.Т. Мнацаканов, С.Н. Юрков. ФТП, 54 (5), $470(2020)$.

[13] M. Lampert, P. Mark. Current injection in solids, (N. Y.London, Academic Press, 1970). J.W. Palmour. Semicond. Sci. Technol., 26, 085016 (2011).
[14] T.T. Mnatsakanov, I.L. Rostovtsev, N.I. Philatov. Solid State Electron., 30, 579 (1987).

[15] T.T. Mnatsakanov. Phys. Status Solidi B, 143, 225 (1987).

Редактор А.Н. Смирнов

\section{Power Schottky-diodes with negative part of differential current-voltage characteristics}

\section{A.G. Tandoev, T.T. Mnatsakanov, S.N. Yurkov}

Moscow Power Engineering Institute, 111250 Moscow, Russia

Abstract It is shown that at high current densities $\left(j>j_{\mathrm{st1}}\right)$ the carrier transport in base layer of Schottky-diodes in addition to commonly accepted diffusive and drift currents is defined by recently discovered diffusion stimulated by quasi-neutral drift (DSQD). The influence of this recently discovered component of current on current-voltage characteristics of Schottky-diode has been investigated. It was shown that in case if the ratio of base width $W$ to ambipolar diffusive length $L$ is higher than 1 $(W / L>1)$ a part with negative differential resistance appears on the current-voltage characteristics of Schottky-diode. The results of analytical investigation are confirmed by numerical calculation using „INVESTIGATION“ program. 